\title{
Clinical Application of a Protocol for Early Diagnosis of Impacted Upper Canines
}

\author{
Hristina Arnautska, DMD, PhD*
}

Assistant Professor, Department of Orthodontics, Faculty of Dental Medicine, Medical University-Varna, Bulgaria

\begin{abstract}
The upper canine is the second most common impacted tooth after lower wisdom teeth. Its disturbed eruption can be diagnosed in early mixed dentition and necessary preventive measures can be taken then. The objective of the present paper is to describe the application of a protocol for early diagnosis of the impaction likelihood of the upper canine and the effects of its application. Compliance with the right sequence of the methodology reported in the proposed protocol will allow dentists to determine whether a patient's canine tooth deviates from its normal path of development and to assess the impaction likelihood of that canine. Given the developmental stage of the dentition at the time of diagnosis and the severity of deviation from the norm, the protocol will guide dentists as to what prevention or treatment steps to undertake.
\end{abstract}

Keywords: impacted canines, prevention, early diagnosis, maxillary canines

\section{Introduction}

The upper canine is the second most common impacted tooth after lower wisdom teeth.[5,12,14] Its disturbed eruption can be diagnosed in early mixed dentition and necessary preventive measures can be taken then. $[6,11,14]$. Impacted upper canines can affect adjacent structures and most often lead to resorption of the roots of adjacent teeth [7]. Early diagnosis of the altered path of the canine and appropriate preventive actions will simplify and ease orthodontic treatment and reduce adverse side effects.[4]

Sambataro, Taguchi and a number of other authors suggest early extraction of the temporary canine as an early step to alter the path of the permanent canine $[6,13,14]$ Kurol et al. monitored the changes in the permanent canine 1 year after extraction of the temporary canine, defining the position and indications for extraction depending on its inclination and angle with the lateral incisor[8]. Taguchi et al. established that when there is an inclination of more than $50^{\circ}$, the tooth is subject to extraction, where as an angulation of about $30^{\circ}$ allows for a good opportunity for withdrawal of the tooth and its introduction into the dental row.[14]
The protocol proposed in this paper involves statistically significant indications for impaction likelihood of upper canines in early mixed dentition and late mixed dentition (1phase)[1,2,3] and depending on the severity of those indicators the protocol suggests different preventive measures.

\section{Aim}

The aim of the present paper is to introduce the application of a protocol for early diagnosis of the impaction likelihood of the upper canine and the effects of its application.

\section{Material and Methods}

The clinical cases described herein are of children with mixed dentition. In order to diagnose the path change of the canines and the need for intervention, the following protocol was used, which combines early mixed dentition (incisors and first molars have erupted) and 1phase of late mixed dentition (lower canines and upper first premolars have erupted): 


\section{International Journal of Science and Research (IJSR) \\ ISSN (Online): 2319-7064}

Index Copernicus Value (2013): 6.14 | Impact Factor (2014): 5.611

Table 1: Protocol for the diagnosis of impaction likelihood of upper canines:

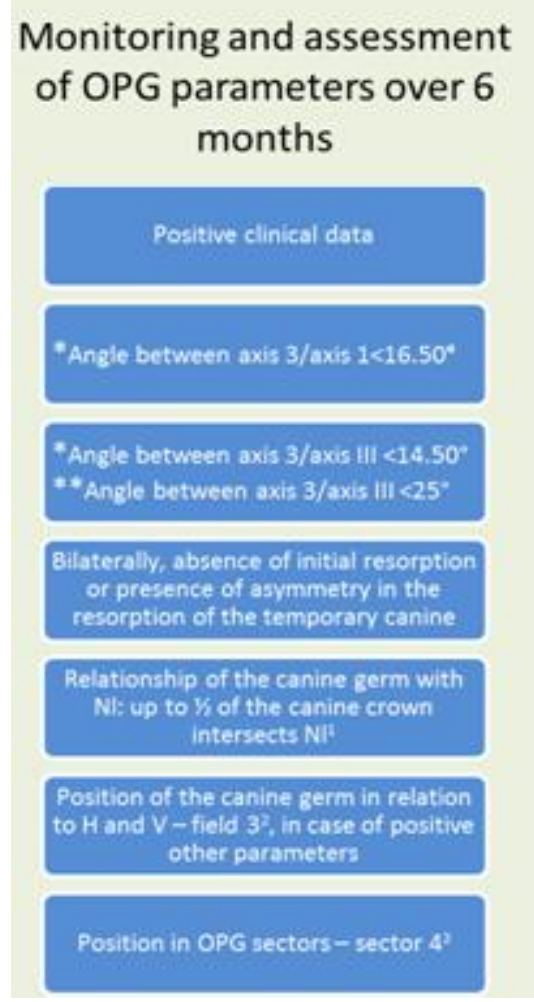

\section{Extraction of III and assessment of OPG} parameters over 6 months
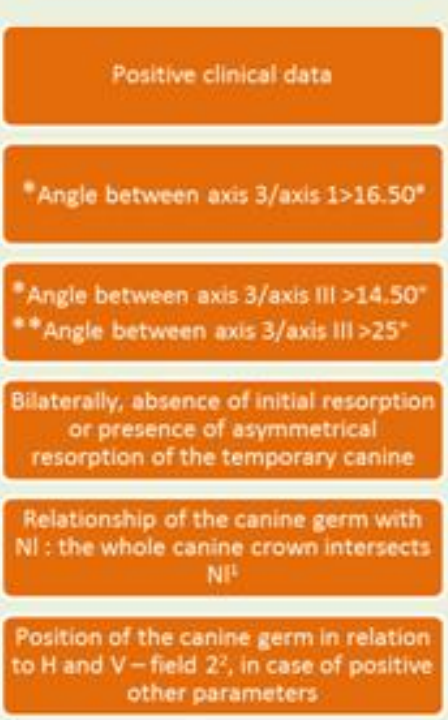

Position in OPG sectors - sectors 3 and $4^{T}$
Extraction of III and treatment and assessment of OPG parameters over 6 months

Angle between axis $3 / a x i s ~ 1>24,40^{\circ}$

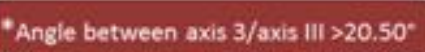
**Angle between axis $3 /$ axis III $>32.25^{\circ}$

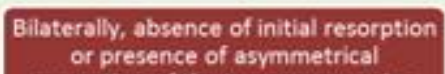

or presence of asymmetrical

resorption of the temporary canine

Relationship of the canine germ with Ni: the whole canine crown and a part of the root intersect $\mathrm{NI}^{\mathrm{I}}$.

Position of the canine germ in relation to $\mathrm{H}$ and $\mathrm{V}$ - field $\mathrm{A}^{\mathrm{2}}$

Position in OPG sectors - sectors 1,2 and $3^{3}$

* - values refer only to early mixed dentition

** - values refer only to 1 phase of late mixed dentition

Application of the protocol involved the use of the following methodology:

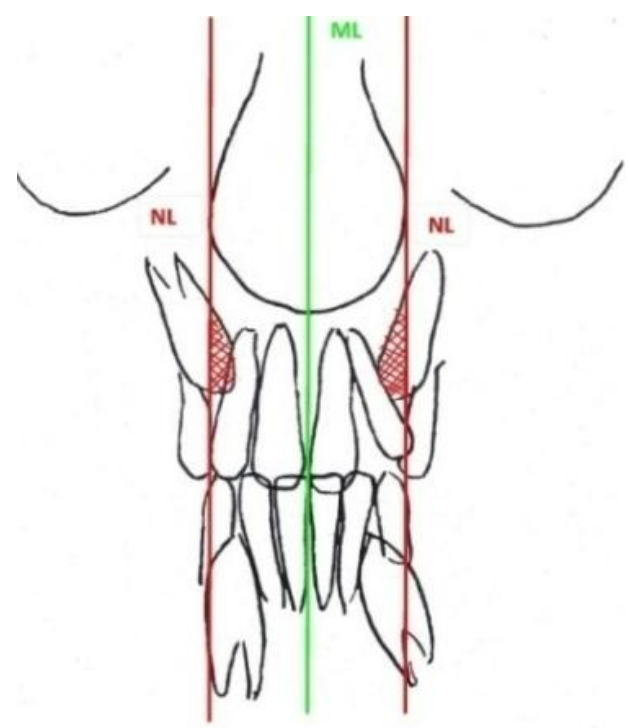

Figure 1: Correlation of the vertical line NL and the of the permanent canine germ

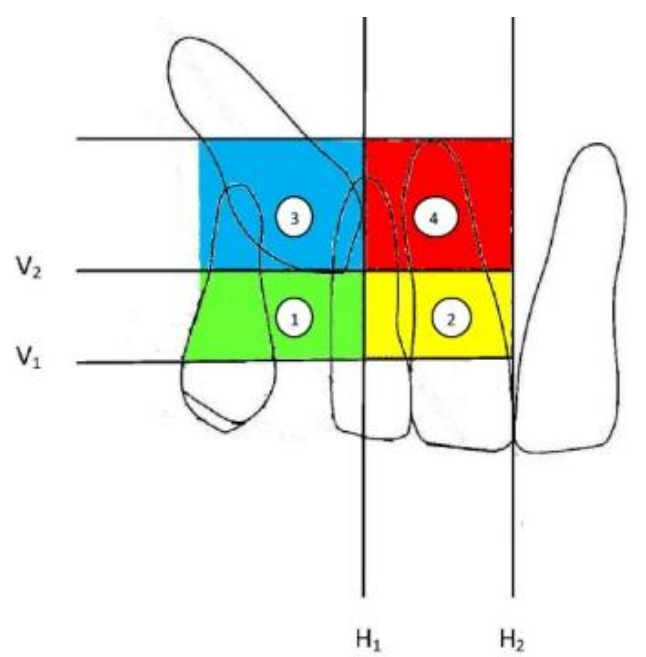

Figure 2: Illustration of canine position as per Zasciurinskiene's methodology ${ }^{(15)}$

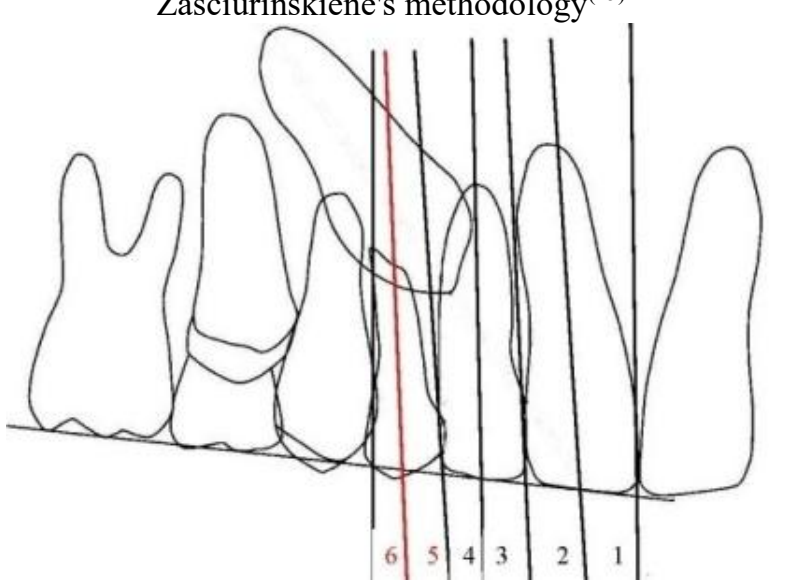

Figure 3: Modified methodology for sector analysis as per crown Ericson\&Kurol 


\section{International Journal of Science and Research (IJSR) \\ ISSN (Online): 2319-7064}

Index Copernicus Value (2013): 6.14 | Impact Factor (2014): 5.611

\section{Case Studies}

The first clinical case is of an 8-year-old boy with early mixed dentition, a strong distal inclination, a Class I patient, as per Angle's classification. (fig.4a) The clinical examination revealed a lack of vestibular convexity of the alveolar ridge on the left over the temporary canine, a strong distal inclination, combined with a marked distovestibular rotation of the left lateral incisor. Angle and sectorindicators in the protocol showedvalues close to the norm. There was agreat deviation in the angle between the inclination of the lateral incisor and the axisof the canine on the right and left. The angle at the left canine, prone to impaction, is 9 degrees greater than the angle at the normal erupting canine on the right. This gave us enough evidence to proceed with the extraction of the temporary left canine. The child was monitored every six months.A year later a follow-up OPG was made and dental impressions were taken. (Figure 4b) As seen in the impression model, there is clearly an improvement in the position of the left lateral incisor, and the X-ray shows visible straightening and descent of the canine tooth, an improved angle between the lateral incisor and the canine as well as closer indicators for both left and right side. As a result, the following conclusion could be drawn: early extraction of the temporary canine led to a change in the eruption path of the permanent canine, close to the norm. The change in the angle values is represented in the Table of Figure $4 \mathrm{~d}$.
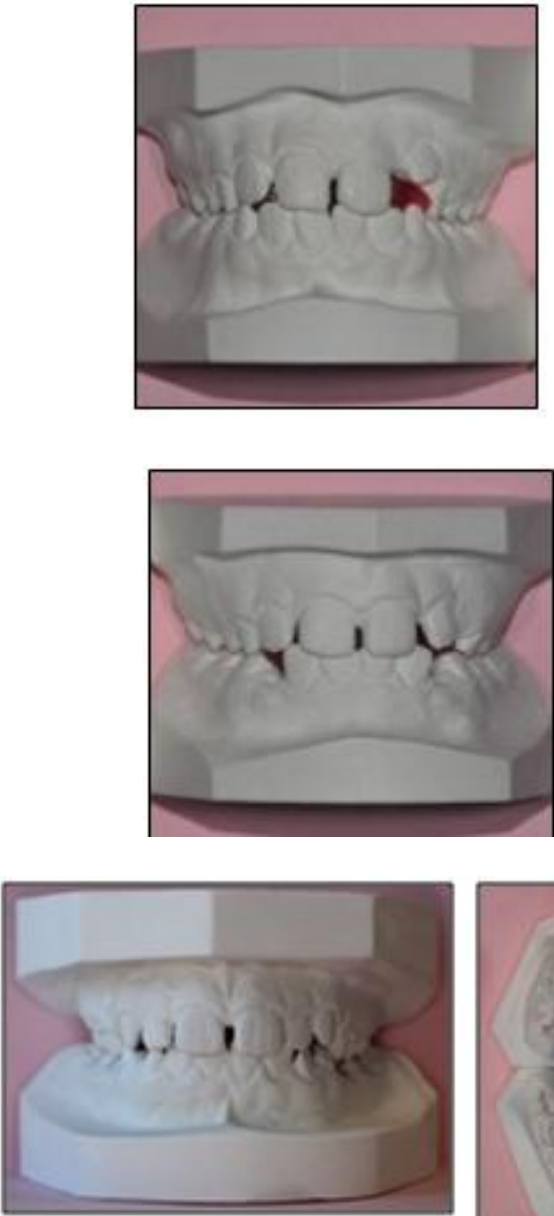

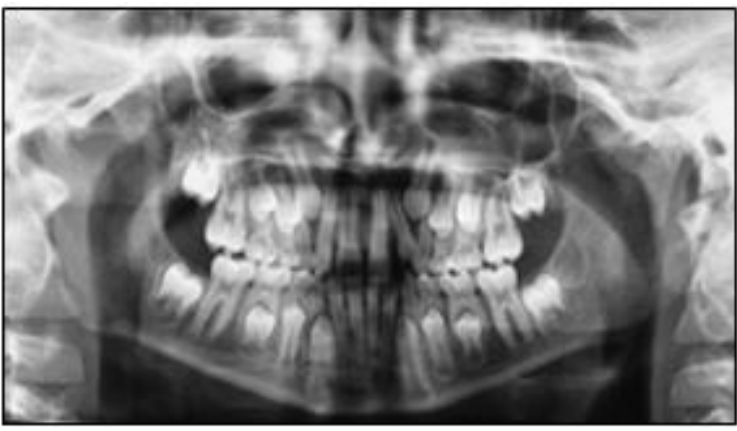

(a)

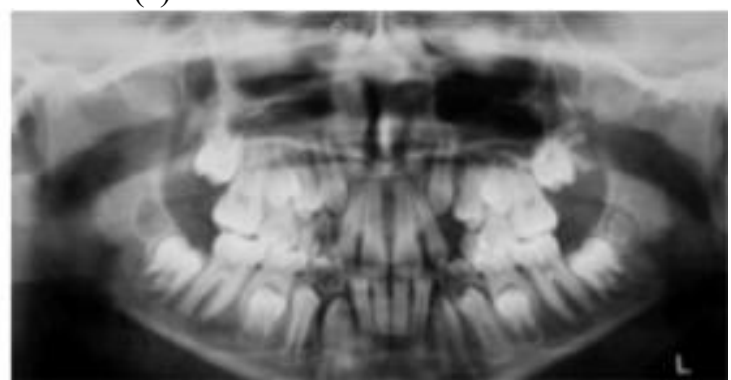

(b)

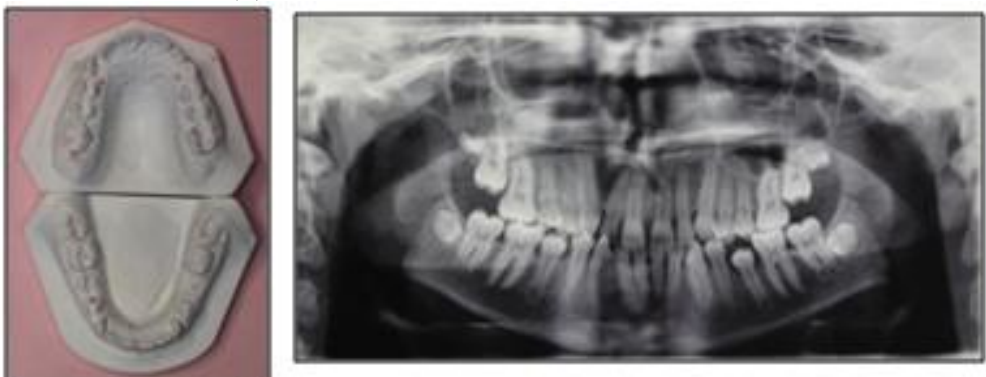

c)

d)

\begin{tabular}{|c|c|c|c|c|c|c|}
\hline \multirow{2}{*}{$\begin{array}{c}\text { Impactors } \\
\text { OPGs }\end{array}$} & \multicolumn{3}{|c|}{ Right canine Norm } & \multicolumn{3}{c|}{ Left canine Impacting } \\
\cline { 2 - 7 } & Angle axis3/axis2 & Angle axis3/axis1 & Angle axis3/axisIII & Angle axis3/axis2 & Angle axis3/axis1 & Angle axis3/axisIII \\
\hline OPG a) & $28^{\circ}$ & $20^{\circ}$ & $11^{\circ}$ & $37^{\circ}$ & $15^{\circ}$ & $11^{\circ}$ \\
\hline OPG b) & $29^{\circ}$ & $14^{\circ}$ & $17^{\circ}$ & $27^{\circ}$ & $13^{\circ}$ & - \\
\hline OPG c) & $18^{\circ}$ & $9^{\circ}$ & - & $12^{\circ}$ & $3^{\circ}$ & - \\
\hline
\end{tabular}

Figure 4: a) plaster models and OPG prior to treatment

b) plaster models and OPG 1 year after extraction of the temporary third tooth

c) plaster models and OPG 2 years after extraction of the temporary third tooth on the left

d) values of angle data on the OPG in different years 


\section{International Journal of Science and Research (IJSR) \\ ISSN (Online): 2319-7064 \\ Index Copernicus Value (2013): 6.14 | Impact Factor (2014): 5.611}

Two years after extraction of the temporary third tooth, the permanent left canine has erupted and is in the desired position (Fig. 4c). In the process of growth malpositions of individual teeth, marked with diastemata, and slight rotations are observed, indicating further need of treatment bythe conventional methodsof orthodontics - correction of mal positions and shaping of the dentalarches.

The second clinical case involved a 10 -year-old boy with delayed resorption and an overall delayed eruption of permanent teeth. The clinical examination revealed microdontia in the left upper lateral incisor, a lack of vestibular convexity of the alveolar ridge over the temporary third tooth on the left, while the right side exhibited well marked convexity. A panoramic radiograph was taken, showing a stronger inclination of the axis of the permanent left canine towards the median line and in relation to the axis of the temporary canine. (Figure 5a) According to sector indicators the left canine was in sector 3 (Figure 2) and sector 4 (Figure 3) which is border-line for patients in that age group. Due to greater inclination value of the canine on the right and the close values, the clinical decision was to

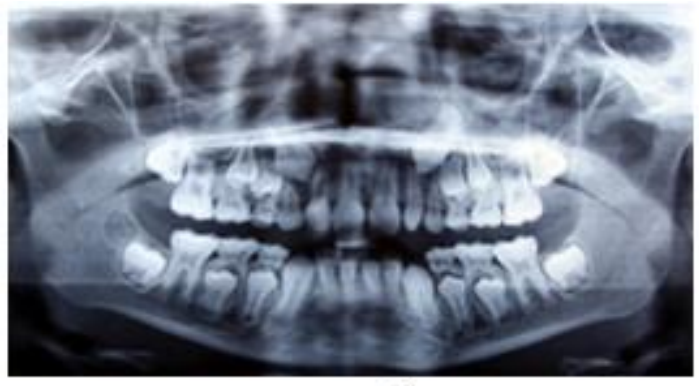

a)

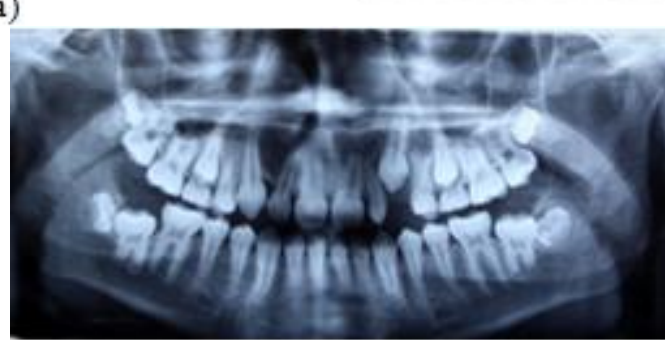

c)

d)

\begin{tabular}{|c|c|c|c|c|c|c|}
\hline \multirow{2}{*}{$\begin{array}{c}\text { Impactors } \\
\text { OPGs }\end{array}$} & \multicolumn{3}{|c|}{ Right canine Norm } & \multicolumn{3}{c|}{ Left canine Impacting } \\
\cline { 2 - 7 } & Angle axis3/ML & Angle axis3/axis1 & Angle axis3/axisIII & Angle axis3/ML & Angle axis3/axis1 & Angle axis3/axisIII \\
\hline OPG a) & $26^{\circ}$ & $28^{\circ}$ & $24^{\circ}$ & $30^{\circ}$ & $15^{\circ}$ & $31^{\circ}$ \\
\hline OPG b) & $26^{\circ}$ & $27^{\circ}$ & $19^{\circ}$ & $33^{\circ}$ & $13^{\circ}$ & $31^{\circ}$ \\
\hline OPG c) & $5^{\circ}$ & $2^{\circ}$ & - & $7^{\circ}$ & $1^{\circ}$ & - \\
\hline
\end{tabular}

Figure 5: a) initial $\mathrm{OPG}$

b) OPG after 1 year

c) OPG 2 years after extraction of the temporary third tooth on the left d) change in angle values on the three OPGs
The third clinical case is of a girl aged 9 years 4 months with early mixed dentition. The patient was brought in due to a concern about a marked change in position of the lateral incisor on the right. The clinical examination revealed vestibular rotation and its distal inclination. On palpation over the area of the right temporary canine, vestibular convexity was missing unlike on the left side. Family history wait and follow the patient. Six months later there was no change in the dental status and the patient was called after six months for another panoramic radiograph. Again following the clinical protocol indicators were taken. Straightening of the right canine tooth and reduction of angle values were established, whereas on the left side the canine had retained its inclination, even a slight deterioration of values was noted. Furthermore, on the right more resorption of the root of the temporary canine was observed, while on the left there was no change in position of the one a year before. (Fig.5b) According to sector indicators the left canine had retained its position, while the values on the right had improved. Based on the analysis of data and the treatment progress following the clinical protocol ( Table 1), extraction of the left temporary canine was undertaken. A removable appliance (a lingual plate) was placed in orderto preserve the space and shaping of the dental arch. Two years after extraction of the temporary canine, the permanent canine descended in the dental arch and its eruption is expected. (Fig. 5c)

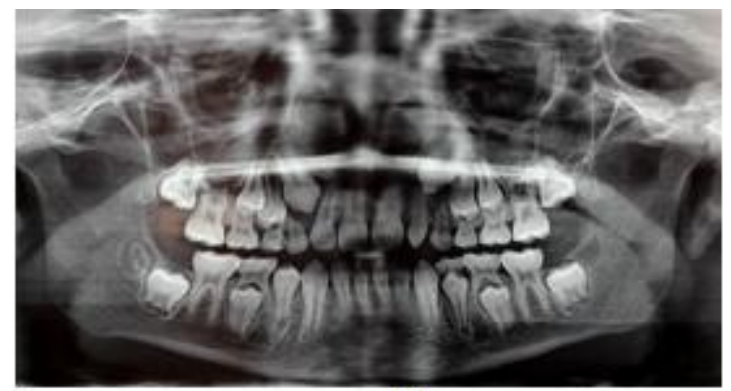

b) revealed that the mother had an impacted canine. The OPG taken (Figure 6a) showed a highly increased inclination of the axis of the right permanent canine in relation to the temporary one and the median line (Table 4a).According to sector allocations the crown of the canine is entirely intercepted by the vertical $\mathrm{Nl}$ (Figure 1), the canine is located in sector 3 (Figure 2) and Sector 4 (Figure 3). These 


\section{International Journal of Science and Research (IJSR) \\ ISSN (Online): 2319-7064 \\ Index Copernicus Value (2013): 6.14 | Impact Factor (2014): 5.611}

indications of the patient referred to the second protocol group. However, we decided to wait for six months to report the changes on the next OPG (Figure 6b). We established a slight improvement in inclination of the canine, but sector indicators were the same; still,sectorallocation hadworsened - the right canine had passed into Sector 3 (Figure 3), which pointed to an impacted canine. Following the clinical protocol (Table 1) the patient was referred for extraction of the temporary third tooth on the right andwas monitored. Nine months after extraction of the temporary canine the panoramic radiograph showed a visible self-correction of the erupting canine. (Figure $6 \mathrm{c}, \mathrm{d}$ )

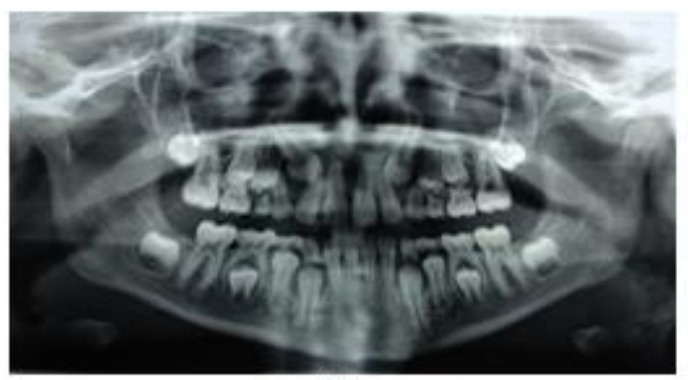

(a)

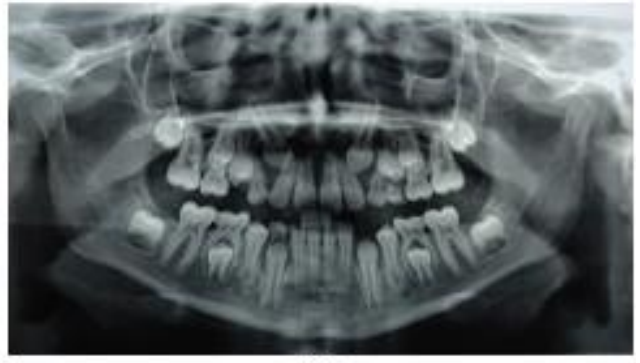

(b)

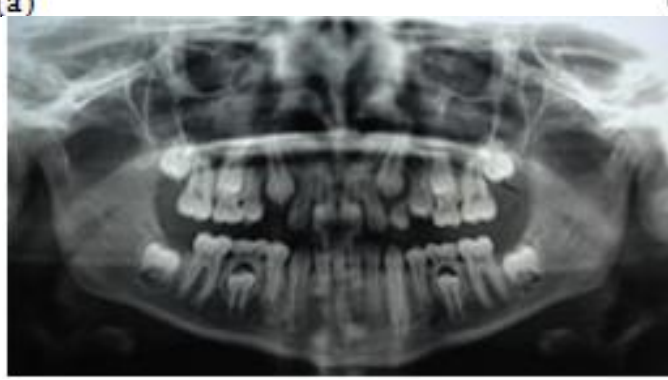

(c)

d)

\begin{tabular}{|c|c|c|c|c|c|c|}
\hline \multirow{2}{*}{$\begin{array}{c}\text { Impactors } \\
\text { OPGs }\end{array}$} & \multicolumn{3}{|c|}{ Right canine Norm } & \multicolumn{3}{c|}{ Left canine Impacting } \\
\cline { 2 - 7 } & Angle axis3/ML & Angle axis3/axis1 & Angle axis3/axisIII & Angle axis3/ML & Angle axis3/axis1 & Angle axis3/axisIII \\
\hline OPG a) & $33^{\circ}$ & $31^{\circ}$ & $23^{\circ}$ & $16^{\circ}$ & $15^{\circ}$ & $13^{\circ}$ \\
\hline OPG b) & $27^{\circ}$ & $25^{\circ}$ & $20^{\circ}$ & $15^{\circ}$ & $16^{\circ}$ & $14^{\circ}$ \\
\hline OPG c) & $10^{\circ}$ & $6^{\circ}$ & - & $13^{\circ}$ & $11^{\circ}$ & $9^{\circ}$ \\
\hline
\end{tabular}

Figure 6 a) initial OPG

b) OPG after 6 months

c) OPG 8 months after extraction of the temporary third tooth on the right

d) проchange in angle values on the three OPGs

The case studies above are an indication that timely extraction of the temporary canine, when there is an early diagnosis of impaction likelihood of the canine, is significant for a change in the eruption path of the permanent canine. In some cases it is sufficient only to extract the temporary canine and to clinically monitor the patient until eruption of the permanent canine $[6,9,11,14]$. In other cases after extraction of the temporary third tooth it is necessary to undertake orthodontic treatment for the purpose of creating additional space for the eruption of the canine.[10,14] In severe cases, where there is a strong mesial inclination, upon extraction of the temporary tooth, straightening of the canine and less mesial inclination are observed. This enables an easier attachment of additional appliances and withdrawal of the canine into the dental arch, reducing the risk of complications. When the canine inclination is over $35^{\circ}$, after extraction of the temporary tooth, it is advisable to wait for straightening of the canine and its spontaneous displacement before undertaking its withdrawal orthodontically.

\section{Conclusion}

The creation of protocols on methods of diagnosis in different stages of the dentition development and their application enables:

1) Compliance with the right sequence of the methodology reported in the proposed protocol, will allow dentists to determine whether a patient's canine tooth deviates from its normal path of development and to assess the impaction likelihood of that canine. Given the developmental stage of the dentition at the time of diagnosis and the severity of deviation from the norm, the protocol will guide dentists as to what prevention or treatment steps to undertake.

2) The protocols created significantly facilitate the approach in diagnosis of impaction likelihood and the time to take prevention or treatment steps with the purpose of altering the path and increasing the eruption likelihood of the incorrectly positioned canine, which in turn shortens treatment.

\section{References}

[1] Arnautska H.,V.KrumovaEarly detection of the tendency to upper canine impaction based on

\section{Volume 4 Issue 11, November 2015}




\section{International Journal of Science and Research (IJSR) \\ ISSN (Online): 2319-7064}

Index Copernicus Value (2013): 6.14 | Impact Factor (2014): 5.611

orthopantomography using sector indicators Orthodontic review, Sofia, 15, 2013, 2, 31-43.

[2] Arnautska H.,V.Krumova Radiographic examination of the position of impacted canines in the upper jawindicators for early diagnostics Orthodontic review, Sofia, 15, 2013, 2, 44-54.

[3] Arnautska H,Krumova V.Protocol for early detection of tendency towards impacted canines in early mixed dentition. Orthodontic review, Sofia, 2014;16(1):6-15.

[4] Becker, A., S. Chaushu.Dental age in maxillary canine ectopia.- Am. J. Orthod. Dentofacial Orthop.,117, 2000, 657-62.

[5] Bishara SE. Impacted maxillary canines: a review.Am J Orthod Dentofacial Orthop.1992;101(2): 159-171.

[6] Ericson S, Kurol J. Early treatment of palatally eruptingmaxillary canines by extraction of the primary canines.Eur J Orthod. 1988;10(4):283-295.

[7] Ericson, S., J. Kurol. Radiographic assessment of maxillary canine eruption in childrenwith clinical signs of eruption disturbance.-Eur. J. Orthod.,8, 1986a, 3, $133-40$.

[8] Kurol,J., EricsonS., AndreasenJ.O.. The impacted maxillary canine.- In: Textbook and color atlas of tooth impaction. 1 ed. J. O. Andreasen, J. K. Petersen, D. M. Laskin,eds. Copenhagen, Munksgaard,1995,125-165.

[9] Litsas G, Acar A. A Review of Early Displaced Maxillary Canines: Etiology, Diagnosis and Interceptive Treatment Open Dent J. 2011; 5: 39-47.

[10] Manne R, Gandikota C, Juvvadi SR, Rama HR, Anche S. Impacted canines: Etiology, diagnosis, and orthodontic management J Pharm Bioallied Sci. 2012 Aug; 4(Suppl 2): 234-238.

[11] Power SM, Short MB. An investigation into the response ofpalatally displaced canines to the removal of deciduous canines and an assessment of factors contributing to favourableeruption. $\mathrm{Br} \quad \mathrm{J}$ Orthod.1993;20(3): 215-223.

[12] Peck, S., L. Peck, M. Kataja. The palatally displaced canineas a dental anomaly of genetic origin.- Angle Orthod., 64, 1994, 4, 249-56.

[13] Sambataro, S., T. Baccetti, L. Franchi, F. Antonini. Early predictivevariables for upper canine impaction as derived frompostero-anterior cephalograms.AngleOrthod.,75, 2004, No 1, 28-34.

[14] Taguchi Y, Kobayashi H, Noda T. A diagnostic proposal to support early treatment of ectopically erupting maxillary canines. Pediatr Dent J. 2005;15(1): 52-57.

[15]Zasciurinskiene, Bjerklin EK, Smailiene D, Sidlauskas A, Puisys A. Initial vertical and horizontal position of palatally impacted maxillary canine and effect on periodontal status following surgical-orthodontic treatment. Angle Orthod. 2008;78(2): 275-280.

\section{Author Profile}

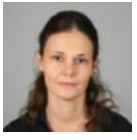

Hristina IvanovaArnautska, DMD, $\mathrm{PhD}$ completed her Master's degree in Dentistry in 1999. She specialized in Orthodontics at the Faculty of Dental Medicine, Medical University - Sofia in 2006, as well as in Health Management at the Medical University - Varna in 2011. Since 2008 she has been an Assistant Professor at the Department of Orthodontics at the Medical University of Varna,
Bulgaria. In 2013 she obtained her PhD degree in Orthodontics and presented her research dissertation "Diagnosis and prediction of canine impaction". Her research interests are in the area of ectopic and impacted canine, preventive orthodontics and interdisciplinary treatment approaches. Dr. Arnautska is a member of WFO, EOS, SIDO, BOS and BSCLO. 